University of Nebraska - Lincoln

DigitalCommons@University of Nebraska - Lincoln

\title{
Thermally Driven Assembly of Nanoparticles in Polymer Matrices
}

Philip J. Costanzo

Army Research Laboratory, Materials DiVision, Aberdeen ProVing Ground, Maryland

Frederick L. Beyer

Army Research Laboratory, Materials DiVision, Aberdeen ProVing Ground, Maryland

Follow this and additional works at: https://digitalcommons.unl.edu/usarmyresearch

Part of the Operations Research, Systems Engineering and Industrial Engineering Commons

Costanzo, Philip J. and Beyer, Frederick L., "Thermally Driven Assembly of Nanoparticles in Polymer Matrices" (2007). US Army Research. 12.

https://digitalcommons.unl.edu/usarmyresearch/12

This Article is brought to you for free and open access by the U.S. Department of Defense at DigitalCommons@University of Nebraska - Lincoln. It has been accepted for inclusion in US Army Research by an authorized administrator of DigitalCommons@University of Nebraska - Lincoln. 


\title{
Thermally Driven Assembly of Nanoparticles in Polymer Matrices
}

\author{
Philip J. Costanzo and Frederick L. Beyer* \\ Army Research Laboratory, Materials Division, Aberdeen Proving Ground, Maryland
}

Received February 20, 2007; Revised Manuscript Received March 29, 2007

\begin{abstract}
Thermally responsive bulk polymer films utilizing reversible Diels-Alder chemistry have been developed. Gold nanoparticles (AuNPs) were passivated with thiol-terminated poly(styrene)- $b$-poly(ethylene glycol) (PS- $b$-PEG) copolymer ligand, where the PS and PEG blocks are joined via a Diels-Alder (DA) linkage. The ligand-functionalized nanoparticles were dispersed within a microphase-separated PS- $b$-poly(methyl methacrylate) (PS- $b$-PMMA) block copolymer. Nanoparticle location was dictated by the compatibility of the external shell with the block copolymer matrix. As cast, the PEG shell compatibilized the nanoparticles with the PMMA domains. Subsequent thermal treatment caused the Diels-Alder linkages between the polymer blocks to dissociate, leaving the AuNPs functionalized by PS ligands. Immiscibility within the PMMA matrix caused AuNP migration to the PS domains. Migration of the Au nanoparticles was determined using morphological characterization via smallangle X-ray scattering (SAXS) and cross-sectional transmission electron microscopy (TEM).
\end{abstract}

\section{Introduction}

Uniform dispersion of additives to a polymer matrix has been extensively studied by a large number of research groups for wide variety of applications. By carefully choosing the additive, one can selectively manipulate specific physical properties including impact strength, magnetic, electronic, and optical properties, and conductivity. For example, by controlling the composition and molecular weight of core-shell polymer nanoparticles, one can manipulate processing parameters for thermoplastic elastomers. ${ }^{1}$ Controlling the addition and dispersion of $\mathrm{CaCO}_{3}$ nanoparticles within PMMA matrices results in abrasion-resistant polymer coatings. ${ }^{2}$ Alumina and silica particles have commonly been utilized to manipulate tensile strength, glass-transition temperatures, and impact strength. ${ }^{3-6}$ Additionally, various computer simulations have been employed to predict not only additive location but the potential impact upon physical property characteristics. ${ }^{7-10}$

Other research groups have focused on selectively altering a polymer surface instead of the bulk morphology. In particular, Koberstein and co-workers have employed selective miscibility to control surface architecture. ${ }^{11-15}$ In those experiments, the key driving force for controlling dispersion was the reduction of surface energy. Using selective miscibility as a method to control additive dispersion and migration, Costanzo et al. functionalized AuNPs with a PS- $b$-PEG copolymer in which the PS and PEG blocks were joined via a Diels-Alder (DA) linkage. ${ }^{16}$ The functionalized nanoparticles were initially dispersed in a PEG matrix, but thermal treatment severed the DA linkages and resulted in the PS-functionalized AuNPs preferentially migrating to the air/film interface. Control experiments conclusively showed that immiscibility of the AuNPs in the polymer matrix dictated particle migration.

Recently, research groups have began to employ block copolymers as templates to control the location of nanoparticle additives within the polymer matrix. ${ }^{17-23}$ In particular, Kramer and co-workers have shown that the location of AuNPs in a PS- $b$-poly(2-vinyl pyridine) (PS- $b$-P2VP) copolymer can be elegantly controlled by two routes. The first, adjusting the ligand

* Correspondence should be addressed to Dr. F. Beyer, Army Research Laboratory, Attn: AMSRD-ARL-WM-MA, Building 4600, Aberdeen Proving Ground, Maryland 21005-5069. E-mail: flbeyer@arl.army.mil. composition from $100 \%$ PS to a 1:1 molar mixture of PS and P2VP ligands, results in the equilibrium location of the AuNPs from the center of the PS domains to the interface between microphase-separated PS and P2VP lamellae. ${ }^{19}$ The second, in which decreasing amounts of only PS-ligand are used, generates the same result. ${ }^{24}$ In both experiments, the location of the AuNPs is determined a priori by ligand composition and coverage, which affect particle size, particle miscibility, and the adsorption energy of the particle at the interface between microphaseseparated domains.

In this work, control over the dispersion of nanoparticle additives is sought both in the as-prepared materials and later, after the application of an external stimulus. Combining the thermal responsiveness of the DA-based PS- $b$-PEG ligand with AuNPs and a PS- $b$-PMMA template should provide control over the initial particle location by the compatibility between the outer, PEG shell of the functionalized Au nanoparticles and the PMMA domains of the microphase-separated block copolymer. Thermal treatment should remove the compatibilizing shell from the particle and render the AuNPs immiscible in the PMMA block. As illustrated in Figure 1, the particles should then migrate at least to the interface between microphase-separated PS and PMMA domains, reducing unfavorable enthalpic interactions. In this manner, therefore, it is hoped that a thermally responsive, hierarchically ordered material can be developed.

\section{Results and Discussion}

Figure 2 illustrates the structure of a gold nanoparticle functionalized with the DA block copolymer and the changes in the ligand that occur with temperature. Below $60{ }^{\circ} \mathrm{C}$, the PEG exterior will dictate miscibility with a polymer matrix. Above $90{ }^{\circ} \mathrm{C}$, cleavage of the DA linkage occurs, severing the PEG shell from the Au nanoparticle, resulting in a PS exterior. The ability to change the miscibility of an additive should allow discrete control of initial additive location and later migration properties. To prove this hypothesis, a PS- $b$-PMMA block copolymer was employed as a templating matrix. After roomtemperature film formation, the PEG shell of the nanoparticle should dictate particle location within the PMMA domains. Subsequent thermal treatment would then result in a PS-coated 

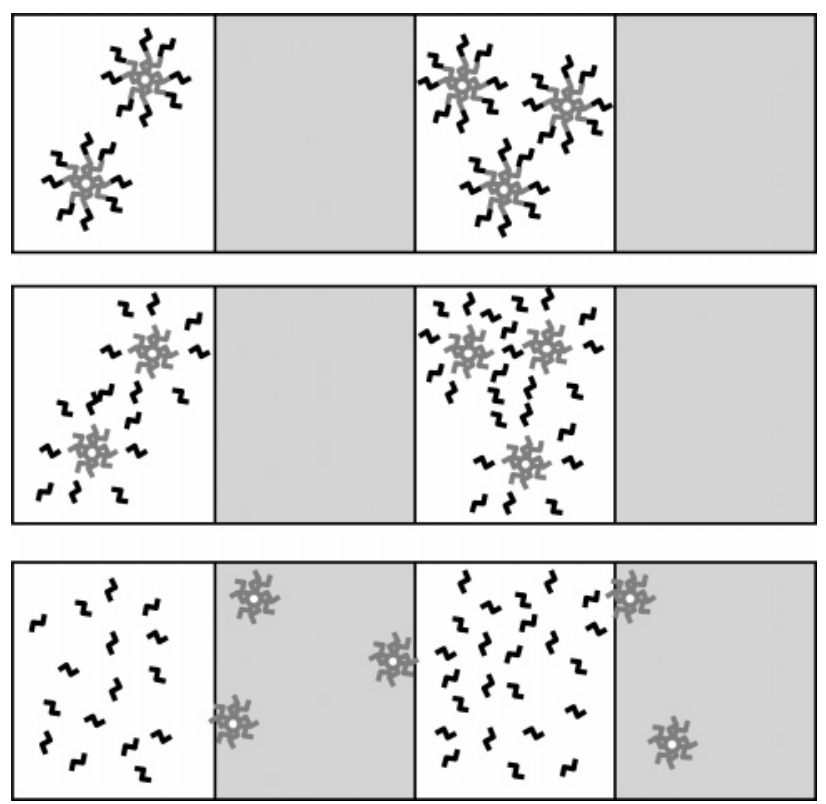

Figure 1. Schematic for particle migration process. (A) Homogeneous distribution of diblock copolymer structure within phase $A$ of $A B$ diblock copolymer. (B) Stimulation of the thin film with $\Delta$, which releases the shell from core. (C) Compatible shell disperses within phase $\mathrm{A}$, while the incompatible core migrates to phase $\mathrm{B}$.

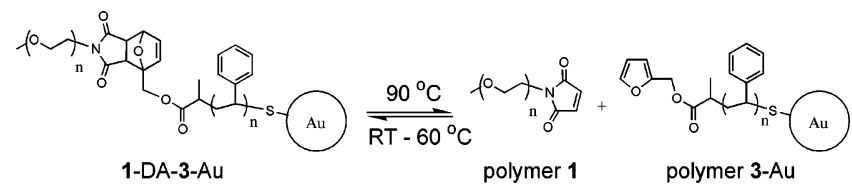

Figure 2. Functionalized Au nanoparticle with thermoresponsive block copolymer ligand based upon Diels-Alder chemistry.

nanoparticle that will migrate to the PS domains to reduce interfacial energy.

Synthesis of the block copolymer-functionalized Au nanoparticles (1-DA-3 Au) has been previously reported. ${ }^{16} \mathrm{Au}$ nanoparticles had a mean diameter of $10 \pm 3 \mathrm{~nm}$ and were functionalized with a $\alpha$-mercapto- $\omega$-furfyl PS linker $\left(M_{\mathrm{n}} 4600\right.$ g/mol; PDI 1.14). A $\alpha$-maleimido- $\omega$-methoxy PEG linker $\left(M_{\mathrm{n}}\right.$ $630 \mathrm{~g} / \mathrm{mol}$; PDI < 1.05) was then utilized in a DA linkage to prepare the DA-functionalized AuNPs. Polymer films were then prepared by dissolving a PS- $b$-PMMA block copolymer in $\mathrm{CH}_{2}-$ $\mathrm{Cl}_{2}$ and adding a stock solution of 1-DA-3-Au to yield various additive loadings. Slow evaporation of solvent-reduced drying defects and resulted in bulk polymer films with thicknesses ranging from 175 to $300 \mu \mathrm{m}$. Additionally, these conditions allowed for phase separation to occur. The films were annealed at $120^{\circ} \mathrm{C}$ for $24 \mathrm{~h}$, then characterized by cross-sectional TEM and SAXS to explore the migration of the functionalized $\mathrm{Au}$ particles within the PS- $b$-PMMA block copolymer matrix. The PS domains were selectively stained with $\mathrm{OsO}_{4}$ vapor, increasing contrast between the PS regions (dark) and the PMMA domains (light). TEM micrographs of the block copolymer materials without DA block copolymer functionalized Au nanoparticles are available in the Supporting Information.

Figure 3 displays cross-sectional TEM micrographs of a PS$b$-PMMA block copolymer $\left(M_{\mathrm{n}} 38 \mathrm{k}-b-37 \mathrm{k}\right.$; PDI $\left.<1.05\right)$ with 5 wt $\%$ of DA-functionalized Au nanoparticles after (A) roomtemperature film formation and (B) annealing at $120{ }^{\circ} \mathrm{C}$ for 24 h. After room-temperature film formation, the DA block copolymer functionalized Au nanoparticles should be located within the PMMA domains. Immiscibility of the PEG-functionalized particles in the PS domains and their miscibility in

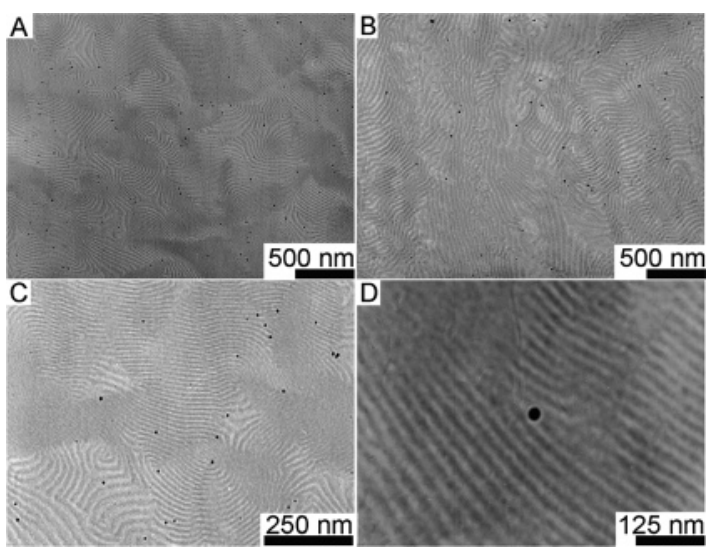

Figure 3. Cross-sectional TEM micrographs of a PS- $b$-PMMA block copolymer $\left(M_{\mathrm{n}} 38 \mathrm{k}-b-37 \mathrm{k}\right.$, PDI $\left.<1.05\right)$ with 5 wt $\%$ of DAfunctionalized $\mathrm{Au}$ nanoparticle additive after RT film formation (A, $\mathrm{C}$, and D) and annealing at $120^{\circ} \mathrm{C}$ for $24 \mathrm{~h}(\mathrm{~B})$. Micrographs (C) and (D) exemplify defects caused by the addition of nanoparticles. After annealing, approximately $82 \%$ of the AuNPs were in the correct phase.

PMMA provide a strong driving force for the particles to preferentially segregate to PMMA. The Flory-Huggins binary segmental mixing parameter for PS and PEG, $\chi_{\mathrm{PEG} / \mathrm{PS}}$, is 0.0644 at room temperature, while $\chi_{\mathrm{PEG} / \mathrm{PMMA}}$ is -0.005 at room temperature. ${ }^{25,26}$ After thermal treatment, the Au nanoparticles should migrate to the PS domains because of the loss of the PEG blocks from the ligands. Final particle location within the PS domains will be dictated by the immiscibility of the PS ligand shell on the nanoparticles and the PMMA domains; at $90^{\circ} \mathrm{C}$, $\chi_{\text {PS/PMMA }}$ is approximately $0.0183 .{ }^{27}$ Although each micrograph may contain only 20-30 particles, at least 170 particles were counted for each sample to provide statistically representative data.

Room-temperature film formation resulted in a homogeneous dispersion of $\mathrm{Au}$ nanoparticles within the PS- $b$-PMMA block copolymer. No selectivity was observed as the ratio of particles located in the correct phase to the incorrect phase is 22 to 25 , or approximately $47 \%$. The lack of selectivity may be explained by the size of the particles relative to the size of the microphaseseparated block copolymer domains. Inclusion of a particle within a polymer domain would require significant polymer chain rearrangement as the particle diameter, approximately 10 $\mathrm{nm}$, is similar to the size of the PS and PMMA domains, approximately $15 \mathrm{~nm}$ each, increasing chain stretching and decreasing conformational entropy. A previous model study supports this hypothesis in addition to observed experimental data. ${ }^{8,23}$ At room temperature, the interfacial energy between the PS and PMMA domains may be greater than the energy required to include the particles within the domains, which would cause the nanoparticles to be excluded from the matrix and act as point defects. In addition, the room-temperature film formation will kinetically trap the block copolymer in a nonequilibrium state, which will reduce the likelihood of correct particle location. Last, the molecular weight of the PEG shell, approximately $630 \mathrm{~g} / \mathrm{mol}$, may not be large enough to demand efficient phase separation. As $\chi$ has dependence upon the molecular weight of the respective blocks, a low-molecularweight shell may lower $\chi$ and reduce the main driving force in controlling the initial particle location. ${ }^{25}$

Annealing the films at $120^{\circ} \mathrm{C}$ for $24 \mathrm{~h}$ cleaves the PEG shell from the nanoparticle, resulting in PS exterior, driving the particles to phase separate from the PMMA domains to reduce interfacial energy. After annealing, $82 \%$ of the particles are 


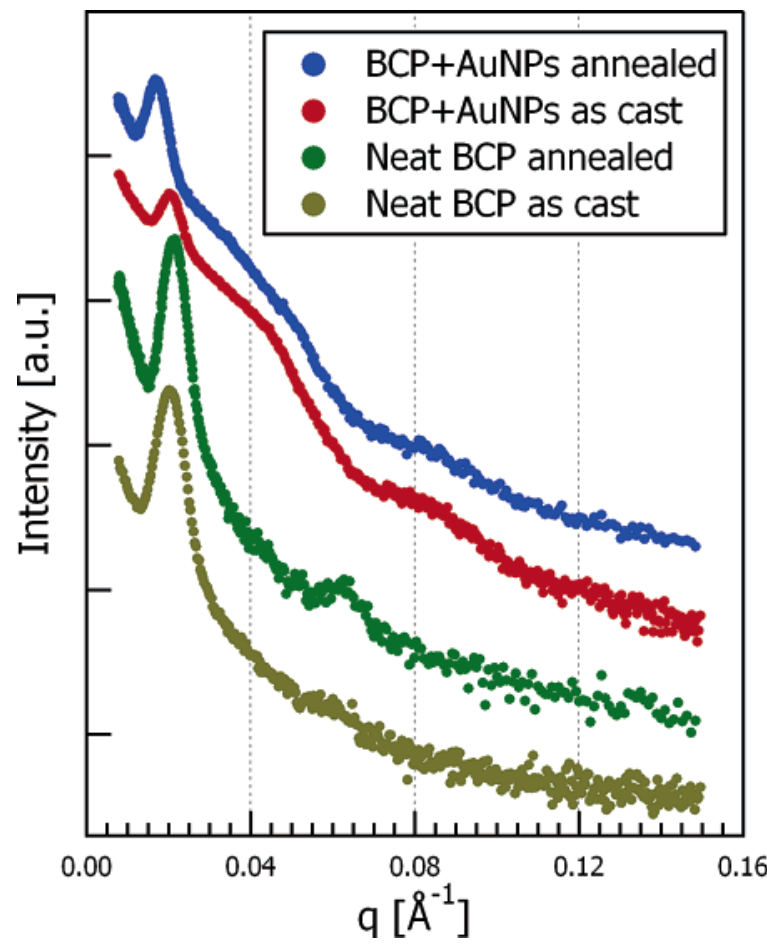

Figure 4. SAXS data for a PS- $b$-PMMA block copolymer $\left(M_{\mathrm{n}} 38 \mathrm{k}-\right.$ $b$-37k, PDI < 1.05) after RT film formation (army green) and $120^{\circ} \mathrm{C}$ anneal for $24 \mathrm{~h}$ (green). Data for PS- $b$-PMMA block copolymer with $5 \mathrm{wt} \%$ of DA-functionalized Au nanoparticle additive after RT film formation (red) and $120{ }^{\circ} \mathrm{C}$ anneal for $24 \mathrm{~h}$ (blue). Note: Data have been offset for clarity. Additionally, not all data points are shown.

located in the correct phase (46 to 10), indicating significant migration occurred. Additionally, the PS domains appear to have increased in width.

Figure 4 displays SAXS data for the PS- $b$-PMMA copolymer both with and without the Au nanoparticle additive. For the neat block copolymer after room-temperature film formation, a single Bragg peak diffraction at $q=0.0201 \AA^{-1}$ is observed, corresponding to a real-space dimension of $31.2 \mathrm{~nm}$. Annealing the neat block copolymer at $120{ }^{\circ} \mathrm{C}$ for $24 \mathrm{~h}$ results in a sharpening of the primary Bragg peak and a slight decrease in domain size to $29.4 \mathrm{~nm}$. A second Bragg reflection is observed at $q=0.0608 \AA^{-1}$, indicating long-range order typical of a symmetric lamellar morphology. (For a lamellar morphology, Bragg reflections occur at integer multiples of the primary scattering maximum; alternating lamellae of equal thicknesses result in the systematic absence of the even Bragg reflections. ${ }^{28}$ ) For the PS- $b$-PMMA block copolymers containing the DAfunctionalized AuNPs, the as-cast material displays a primary Bragg maximum corresponding to a domain size of $31.3 \mathrm{~nm}$, consistent with the neat block copolymer after room-temperature film formation.

Scattering from the AuNPs is also evident. As discussed previously, ${ }^{16}$ the contribution to the scattered intensity from the form factor for $10 \mathrm{~nm}$ spherical particles appears beginning around $q \approx 0.03 \AA^{-1}$ and includes the Guinier "knee" and one additional fringe at $q \approx 0.08 \AA^{-1}$. The strong contribution to the scattering data from the AuNPs masks the effect of incorporating the AuNPs on the long-range order of the block copolymer matrix. However, comparison of the micrographs in Figure 3 with those for the neat block copolymer given in the Supporting Information indicate that the long-range order is moderately diminished as a result of nanoparticle addition.

Annealing the same sample at $120{ }^{\circ} \mathrm{C}$ for $24 \mathrm{~h}$ results in a significant increase in domain size, to $37.6 \mathrm{~nm}$, which is

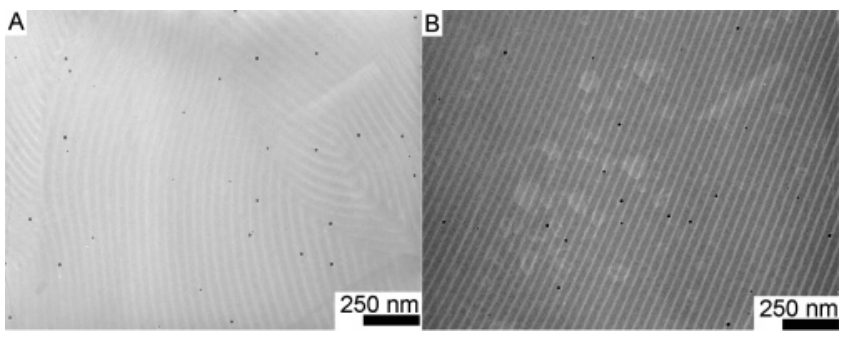

Figure 5. Cross-sectional TEM micrographs of a PS- $b$-PMMA block copolymer $\left(M_{\mathrm{n}} 90 \mathrm{k}-b-90 \mathrm{k}\right.$, PDI $\left.<1.05\right)$ with 5 wt $\%$ of DAfunctionalized Au nanoparticle additive after (A) RT film formation; (B) annealing at $120^{\circ} \mathrm{C}$ for $24 \mathrm{~h}$. After annealing, approximately $88 \%$ of the AuNPs were in the correct phase.

consistent with the increase in PS domain thickness noted in Figure 3. This increase in domain spacing indicates that the particles are being incorporated into the lamellar morphology where previously they were excluded, rather than simply moving from one lamella to another. In the latter case, the total volume of the lamellar period would not change because volume removed from one phase would be added to the other. Here, the increase in period indicates a corresponding increase in total volume, which can only occur with the addition of volume to the morphology, as has been demonstrated by blending homopolymers or even nanoparticles with block copolymers. ${ }^{21,29,30}$ The nanoparticles initially serve as defect points, which can be observed in the TEM micrographs as multiple domains, are evident and exist without long-range order. In particular, parts $\mathrm{C}$ and D of Figure 3 have been selected to focus on the correlation between particle location and the abundance of grain boundaries and defects. Additionally, recent work from Bockstaller et al. demonstrated that grain boundaries and defects can be stabilized by nanoparticle additives. ${ }^{31}$

As previously noted, the relationship between particle size and polymer domain size is likely to effect initial particle location and selectivity within the block copolymer template. In the first set of samples, the particles were large relative to the size of the microphase-separated domains. If the particles were small relative the domain size, the entropic penalty for incorporating the particles into the microphase-separated domains would likely be offset by the enthalpic gains and reduction of surface energy. To further probe this hypothesis, a PS- $b$ PMMA block copolymer matrix with higher molecular weight was employed. Increasing the molecular weight of the block copolymer template should increase domain size and should improve the selectively of the initial particle location.

Figure 5 shows representative cross-sectional TEM micrographs of the PS- $b$-PMMA block copolymer $\left(M_{\mathrm{n}} 90 \mathrm{k}-b\right.$ - $90 \mathrm{k}$; PDI < 1.05) with 5 wt $\%$ of DA-functionalized Au nanoparticle additive after (A) room-temperature film formation and (B) annealing at $120{ }^{\circ} \mathrm{C}$ for $24 \mathrm{~h}$. After room-temperature film formation, $54 \%$ (20 to 17) of the particles were located in the correct phase (PMMA), a modest improvement over the lowermolecular-weight block copolymer matrix. Annealing the films at $120{ }^{\circ} \mathrm{C}$ for $24 \mathrm{~h}$ results in significant and more efficient particle migration, with $88 \%$ (22 to 3 ) of the particles in the correct phase (PS). This increase in segregation efficiency supports the argument that the relative size of the nanoparticle is an important factor in the degree to which particles are located in the domain in which they are miscible.

SAXS data for larger-molecular-weight PS- $b$-PMMA block copolymer matrix containing functionalized AuNPs is shown in Figure 6. Characterization of the neat PS- $b$-PMMA block copolymer after room-temperature film formation yields Bragg reflections at $q=0.0101 \AA^{-1}$ and $0.0304 \AA^{-1}$, corresponding 


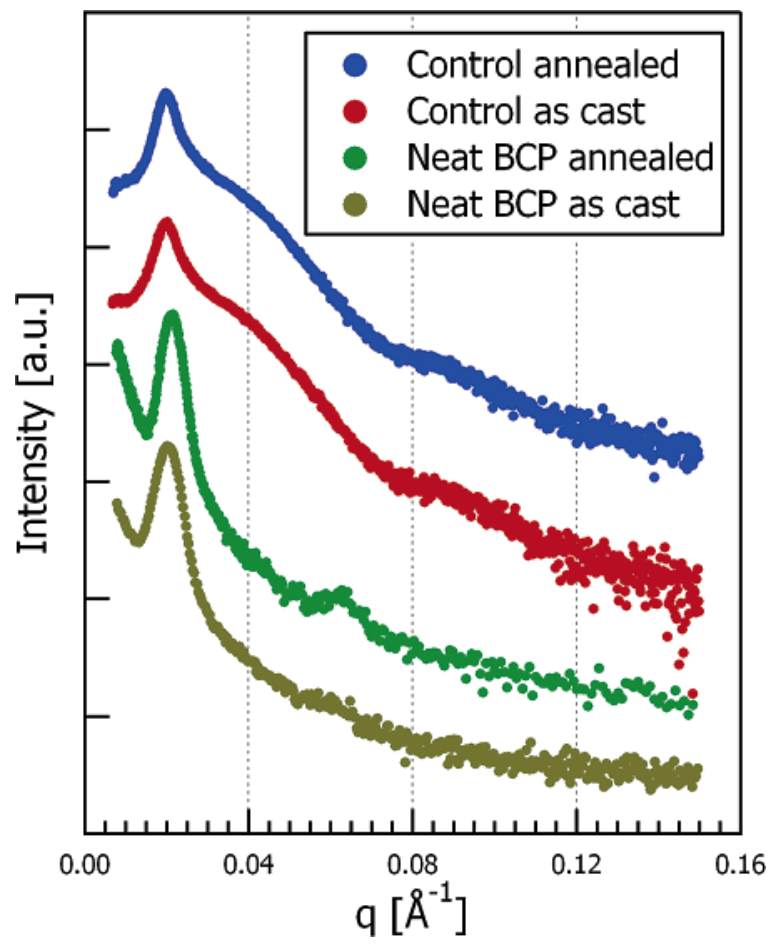

Figure 6. SAXS data for a PS- $b$-PMMA block copolymer $\left(M_{\mathrm{n}} 90 \mathrm{k}-\right.$ $b$-90k, PDI < 1.05) after RT film formation (army green) and $120{ }^{\circ} \mathrm{C}$ anneal for $24 \mathrm{~h}$ (green). Data for PS- $b$-PMMA block copolymer with $5 \mathrm{wt} \%$ of DA-functionalized Au nanoparticle additive after RT film formation (red) and $120{ }^{\circ} \mathrm{C}$ annealing for $24 \mathrm{~h}$ (blue). Data have been offset for clarity.

to a lamellar period of approximately $62.2 \mathrm{~nm}$. Annealing the block copolymer at $120{ }^{\circ} \mathrm{C}$ for $24 \mathrm{~h}$ results in a sharpening of the primary Bragg maximum and the appearance of a third Bragg reflection at $q=0.0525 \AA^{-1}$. The observation of the fifth order Bragg reflection indicates a very well-ordered morphology. The lamellar period decreases slightly to $58.7 \mathrm{~nm}$, a change typical of an annealed block copolymer sample.

Analysis of the PS- $b$-PMMA block copolymer containing the DA-functionalized AuNPs generated from room-temperature film formation yields a Bragg peak corresponding to a domain size of approximately $61.8 \mathrm{~nm}$, consistent with the block copolymer without the additive after room-temperature film formation; however, significant broadening of the peak is also observed, indicating that the nanoparticles disrupt long-range order. Additionally, scattering from the individual AuNPs is again apparent. Annealing the sample at $120{ }^{\circ} \mathrm{C}$ for $24 \mathrm{~h}$ sharpens the Bragg peak, but in contrast to the lower-molecularweight PS- $b$-PMMA block copolymer matrix, no shift is observed, indicating that the majority of AuNPs were incorporated into the lamellar morphology prior to annealing.

Finally, a third block PS- $b$-PMMA block copolymer with a domain size of approximately $110 \mathrm{~nm}$ was utilized as a matrix. Figure 7 shows cross-sectional TEM micrographs of the PS- $b$ PMMA block copolymer $\left(M_{\mathrm{n}} 170 \mathrm{k}-b-168 \mathrm{k}\right.$; PDI < 1.05) with 5 wt $\%$ of DA-functionalized Au nanoparticle additive after (A) room-temperature film formation and (B) annealing at $120^{\circ} \mathrm{C}$ for $24 \mathrm{~h}$. After room-temperature film formation, 62\% (73 to 45) of the functionalized Au nanoparticles are observed in the correct (PMMA) phase, a substantial improvement over the behavior for the two lower-molecular-weight block copolymer matrices. Approximately $90 \%$ (32 to 4) of the particles were observed in the PS domains, the highest efficiency of the three samples characterized.

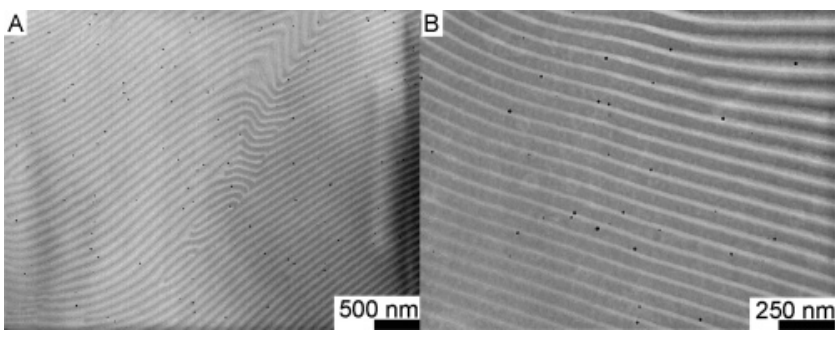

Figure 7. Cross-sectional TEM micrographs of a PS- $b$-PMMA block copolymer $\left(M_{\mathrm{n}} 170 \mathrm{k}-b-168 \mathrm{k}\right.$, PDI $\left.<1.05\right)$ with 5 wt $\%$ of DAfunctionalized Au nanoparticle additive after (A) RT film formation; (B) annealing at $120^{\circ} \mathrm{C}$ for $24 \mathrm{~h}$. After annealing, approximately $90 \%$ of the AuNPs were in the correct phase.

Two primary conclusions may be drawn from the set of data presented above. First, the relative size of the particles has a significant effect on their inclusion in the microphase-separated domains of the block copolymer. For the highest-molecularweight matrix, the particles were easily less than half the size of either the PS or PMMA domains; accordingly, the particles dispersed well and the fraction incorporated into the PS domains after annealing was the highest observed despite the kinetic limitations on particle migration in a high-molecular-weight matrix. However, when the particle size was large relative to the domain size, as for the lowest-molecular-weight block copolymer, the initial dispersion was essentially random and improved only slightly with annealing. As noted above, a large number of particles were observed at defects in the block copolymer morphology, highlighting the limited ability of the microphase-separated polymer matrix to incorporate the particles into the lamellar domains. Moreover, the domain size of the lowest-molecular-weight PS- $b$-PMMA block copolymer $\left(M_{\mathrm{n}}\right.$ $38 \mathrm{k}-b-37 \mathrm{k}$; PDI < 1.05) actually increases after annealing. If the particles were always incorporated into the block copolymer morphology, a change in domain size would not be expected because of incompressibility constraints. If material migrates from one phase to another, then the loss of volume of one phase will be offset by the gain in volume in the other, and no net shift should be observed. Indeed, for the two higher-molecularweight matrices, the lamellar period is unchanged by annealing. An increase in the lamellar period indicates an increase in volume, and because thermal treatment was the only manipulation applied to the sample, one can postulate that the AuNPs are being excluded from the block copolymer at room temperature and included after annealing. Here, the effects of increased chain stretching and decreased conformational entropy outweigh the enthalpic benefits of incorporating the particles into the lamellar domains, resulting in significant exclusion of the particles from the lamellar morphology.

The second significant observation is that, as hypothesized, using ligands incorporating a Diels-Alder linkage allows the dispersion of the nanoparticles to be altered by simply annealing the materials. When annealed at $90{ }^{\circ} \mathrm{C}$, the upper end of the temperature range over which the Diels-Alder linkage dissociates, a significant fraction of the AuNPs migrate from the PMMA domains in which they were originally dispersed to the PS domains. To ensure particle migration was being directed by compatibility between the nanoparticle ligand and polymer matrix, control experiments in which Au nanoparticles were passivated with $\alpha$-methoxy- $\omega$-mercapto PEG polymers were performed. Here, the PEG ligands should direct alignment within the PMMA domains regardless of the processing conditions. Figure 8 depicts cross-sectional TEM micrographs of the PS$b$-PMMA block copolymer $\left(M_{\mathrm{n}} 38 \mathrm{k}-b-37 \mathrm{k}\right.$; PDI $\left.<1.05\right)$ with 7 wt $\%$ of DA-functionalized Au nanoparticle additive after (A) 


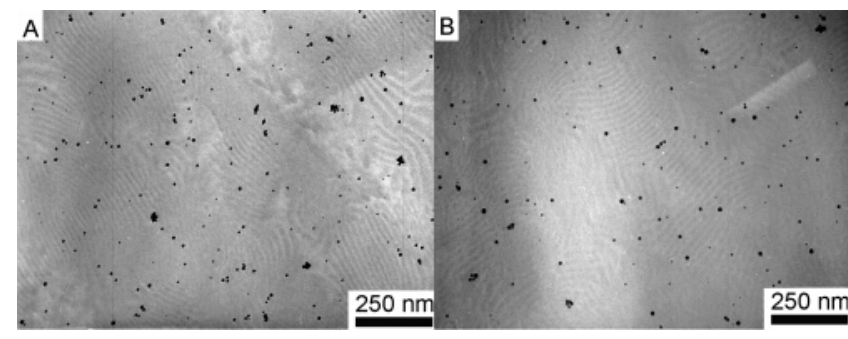

Figure 8. Cross-sectional TEM micrographs of a PS- $b$-PMMA block copolymer $\left(M_{\mathrm{n}} 38 \mathrm{k}-b-37 \mathrm{k}\right.$, PDI $\left.<1.05\right)$ with 7 wt $\%$ of PEGfunctionalized $\mathrm{Au}$ nanoparticle additive after (A) RT film formation; (B) annealing at $120^{\circ} \mathrm{C}$ for $24 \mathrm{~h}$. After annealing, approximately $55 \%$ of the AuNPs were in the correct phase.

room-temperature film formation and (B) annealing at $120^{\circ} \mathrm{C}$ for $24 \mathrm{~h}$. Again, after room-temperature film formation, a homogeneous dispersion of particles within the PS- $b$-PMMA block copolymer is observed. An improvement is observed for initial selectivity as the ratio of particles located in the correct phase to the incorrect phase is 123 to $111, \sim 53 \%$. The larger molecular weight of $\alpha$-methoxy- $\omega$-mercapto PEG ligands compared to the PEG shell employed for the DA-functionalized Au nanoparticles, 5000 vs $630 \mathrm{~g} / \mathrm{mol}$, respectively, increases phase separation. Overall, the selectivity is still limited due to the domain size and film-processing conditions.

One would expect annealing the sample to increase longrange order and increase particle selectivity; however, this is not observed. After annealing the sample at $120{ }^{\circ} \mathrm{C}$ for $24 \mathrm{~h}$, minimal improvement upon the selectivity is observed as the ratio of particles within the correct phase to incorrect phase is 89 to 74 , approximately $55 \%$. As previously mentioned, thermal treatment of the functionalized AuNPs releases a low-molecularweight PEG polymer into the matrix, which can plasticize the matrix and improve particle mobility. The PEG-functionalized Au nanoparticles do not release a plasticizing agent to improve particle mobility. Without significant particle migration, the polymer domains do not swell, as evident from the TEM micrographs and SAXS analysis, Figure 9.

\section{Conclusion}

Gold nanoparticles with a mean diameter of $10 \mathrm{~nm}$ were passivated with PS- $b$-PEG block copolymer ligands in which the PS and PEG blocks were joined via a Diels-Alder (DA) linkage. Using PS- $b$-PMMA block copolymers as a templating matrix, the DA-functionalized AuNPs were dispersed within the block copolymers. The location of the particles in the as-cast films was determined by interactions between the ligand shell of the AuNPs and the PS- $b$-PMMA copolymer and the size disparity between the nanoparticles and the lamellar domains of the microphase-separated matrix. Where the particle diameter was comparable to the lamellar thickness, the AuNPs were randomly dispersed with no preference for either the PS or PMMA domains in the as-cast bulk films. After annealing, a majority of the AuNPs were found either in the PS domains or at the interfaces between the PS and PMMA domains. Annealing cleaves the PEG from the Au nanoparticle, creating a PSfunctionalized nanoparticle exterior. The AuNPs then migrated to the PS phase to reduce interfacial energy. Particle location and migration was dependent upon the composition and molecular weight of the ligands employed. Additionally, a swelling of the polymer domains was observed when a significant number of functionalized $\mathrm{Au}$ nanoparticles were localized within a single domain. For a higher molecular weight PS- $b$-PMMA copolymer matrix, for which the lamellar mor-

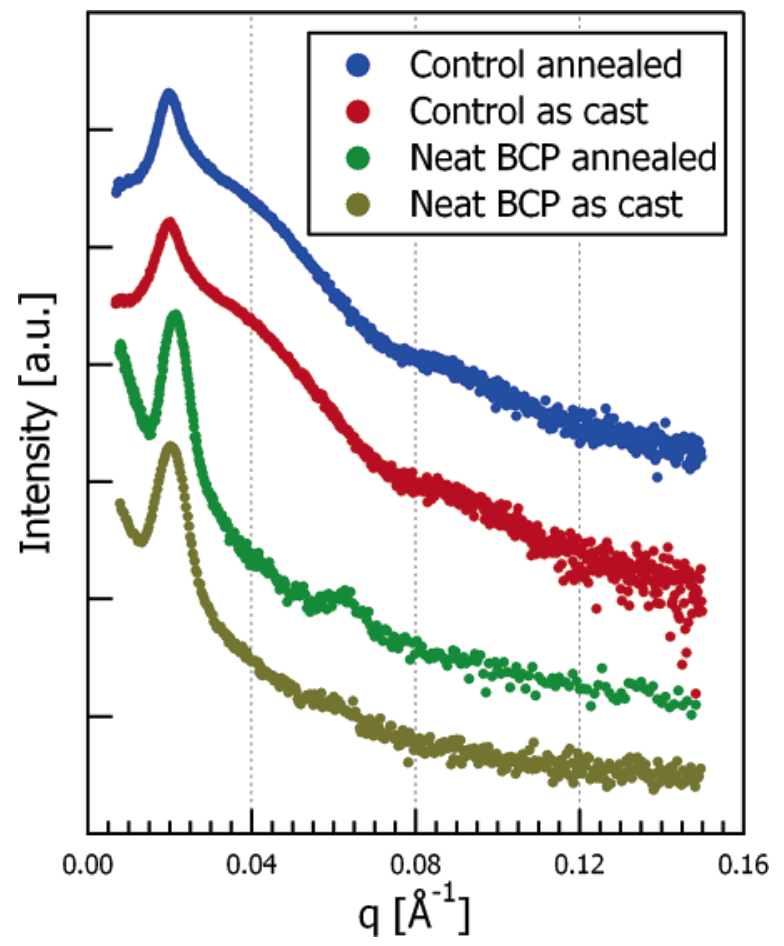

Figure 9. SAXS data for a PS- $b$-PMMA block copolymer $\left(M_{\mathrm{n}} 38 \mathrm{k}-\right.$ $b$-37k, PDI < 1.05) after RT film formation (army green) and $120^{\circ} \mathrm{C}$ anneal for $24 \mathrm{~h}$ (green). Data for PS- $b$-PMMA block copolymer with $7 \mathrm{wt} \%$ of PEG-functionalized Au nanoparticle additive after RT film formation (red) and $120{ }^{\circ} \mathrm{C}$ annealing for $24 \mathrm{~h}$ (blue). Data have been offset for clarity.

phology has a period of $\sim 110 \mathrm{~nm}$, the as-cast materials show a significant improvement in the location of the PS-b-PEG ligand passivated AuNPs, which also subsequently migrate to the PS domains upon annealing. Control experiments verified that the PS-ligand fragment left on the particle after the DielsAlder linkage is broken is required for particle migration.

\section{Experimental}

Materials. All materials were purchased from commercial sources and used without further purification. Au nanoparticles functionalized with PEG- $b$-PS block copolymers containing DA linkages were prepared as previously reported. ${ }^{16}$

Instrumentation and Analysis. Bulk films were prepared by slow solvent evaporation from dilute solutions of PS- $b$-PMMA copolymers with various wt \% loadings of functionalized $\mathrm{Au}$ nanoparticles in dichloromethane. Bulk films were embedded in EpoFix, epoxy-based resin, which cures at room temperature. Specimens for transmission electron microscopy (TEM) were prepared by cutting sections approximately $30 \mathrm{~nm}$ thick using a Leica Ultracut UCT microtome and a diamond knife utilizing $\mathrm{H}_{2} \mathrm{O}$ was a trough liquid. Sections were collected upon Formvar-coated TEM grids and stained with $\mathrm{OsO}_{4}$ for $1 \mathrm{~h}$. Bright-field TEM data were obtained using JEOL 200CX operated at $120 \mathrm{kV}$ accelerating voltage. Small-angle X-ray scattering (SAXS) data were collected using the Army Research Laboratory SAXS instrument, which consists of a Rigaku Ultrax 18 rotating $\mathrm{Cu}$ anode $\mathrm{X}$-ray generator (operated at $45 \mathrm{kV}, 100 \mathrm{~mA}$ ), a customized $3 \mathrm{~m}$ three-pinholecollimated camera, and a Molecular Metrology multiwire area detector. Characteristic $\mathrm{Cu} \mathrm{K} \alpha \mathrm{X}$-rays were selected using a $\mathrm{Ni}$ foil filter $(\lambda=1.542 \AA)$. The sample-to-detector distance was approximately $1.5 \mathrm{~m}$. The raw $2 \mathrm{D}$ data were corrected for background and detector noise prior to azimuthally averaging. Corrected 1D data are presented as intensity, $I(q)$, as a function of the magnitude of the scattering vector, $q$, where $q=4 \pi \cdot \sin (\theta) / \lambda$, $2 \theta$ is the scattering angle, and $\lambda$ is the $\mathrm{X}$-ray wavelength. Absolute scaling was achieved using type- 2 glassy carbon as a secondary 
standard. ${ }^{32}$ All data correction and analysis were performed using Wavemetrics Igor Pro v. 5.04B.

Acknowledgment. Funding was provided by the Army Research Laboratory (ARL). This research was also supported in part by an appointment to the Research Participation Program at the U.S. Army Research Laboratory administered by the National Research Council through an interagency agreement between the U.S. Department of Energy and USARL. Dr. Kris Stokes (SOAN Labs) and Dr. Josh Orlicki (ARL) are acknowledged for helpful discussions.

Supporting Information Available: Original TEM micrographs. This material is available free of charge via the Internet at http://pubs.acs.org.

\section{References and Notes}

(1) Gonzalez-Leon, J. A.; Ryu, S.-W.; Hewlett, S. H.; Ibrahim, S. H.; Mayes, A. M. Macromolecules 2005, 38, 8036-8044.

(2) Avella, M.; Errico, M. E.; Martuscelli, E. Nano Lett. 2001, 1, $213-$ 217.

(3) Ash, B. J.; Siegel, R. W.; Schadler, L. S. Macromolecules 2004, 37, $1358-1369$

(4) Sumita, M.; Tsukihi, H.; Miyasaka, K.; Ishikawa, K. J. Appl. Polym. Sci. 1984, 29, 1523-1530.

(5) Ou, Y.; Yang, F.; Yu, Z.-Z. J. Polym. Sci., Part B: Polym. Phys. 1998, 36, 789-795.

(6) Petrovic, Z. S.; Zhang, W. Mater. Sci. Forum 2000, 352, 171-176.

(7) Schultz, A. J.; Hall, C. K.; Genzer, J. Macromolecules 2005, 38, 30073016.

(8) Huh, J.; Ginzburg, V. V.; Balazs, A. C. Macromolecules 2000, 33, 8085-8096.

(9) Thompson, R. B.; Ginzburg, V. V.; Matsen, M. W.; Balazs, A. C. Science 2001, 292, 2469-2472.

(10) Thompson, R. B.; Jasnow, D.; Matsen, M. W.; Balazs, A. C. Macromolecules 2002, 35, 1060-1071.

(11) O'Rourke-Muisener, P. A. V.; Koberstein, J. T.; Kumar, S. Macromolecules 2003, 36, 771-781.

(12) O'Rourke-Muisener, P. A. V.; Jalber, C. A.; Yuan, C.; Baetzold, J.; Mason, R.; Wong, D.; Kim, Y. J.; Koberstein, J. T. Macromolecules 2003, 36, 2956-2966.
(13) Jalbert, C. A.; Koberstein, J. T.; Hariharan, A.; Kumar, S. Macromolecules 1997, 30, 4481-4490.

(14) Mason, R.; Jalbert, C. J.; O'Rourke-Muisener, P. A. V.; Koberstein, J. T. Adv. Colloid Interface Sci. 2001, 94, 1.

(15) Yuan, C.; Ouyang, M.; Koberstein, J. T. Macromolecules 1999, 32, 2329-2333.

(16) Costanzo, P. J.; Demaree, J. D.; Beyer, F. L. Langmuir 2006, 22 10251-10257

(17) Binder, W. H.; Kluger, C.; Straif, C. J.; Friedbacher, G. Macromolecules 2005, 38, 9405-9410.

(18) Bockstaller, M. R.; Lapetnikov, Y.; Margel, S.; Thomas, E. L. J. Am. Chem. Soc 2003, 125, 5276-5277.

(19) Chiu, J. J.; Kim, B. J.; Kramer, E. J.; Pine, D. J. J. Am. Chem. Soc 2005, 127, 5036-5037.

(20) Gupta, S.; Zhang, Q. L.; Emrick, T.; Balazs, A. C.; Russell, T. P. Nat. Mater. 2006, 5, 229-233.

(21) Kim, B. J.; Chiu, J. J.; Yi, G. R.; Pine, D. J.; Kramer, E. J. Adv. Mater $\mathbf{2 0 0 5}, 17,2681$.

(22) Laicer, C. S. T.; Chastek, T. Q.; Logde, T. P.; Taton, T. A. Macromolecules 2005, 38, 9749-9756.

(23) Bockstaller, M. R.; Mickiewicz, R. A.; Thomas, E. L. Adv. Mater. 2005, 17, 1331-1349.

(24) Kim, B. J.; Bang, J.; Hawker, C. J.; Kramer, E. J. Macromolecules 2006, 39, 4108-4114

(25) Zhu, L.; Cheng, S. Z. D.; Calhoun, B. H.; Ge, Q; Quirk, R. P Thomas, E. L.; Hsiao, B. S.; Yeh, F.; Lotz, B. Polymer 2001, 42, $5829-5839$.

(26) Sato, T.; Katayama, K.; Suzuki, T.; Shiomi, T. Polymer 1998, 39, $773-780$.

(27) Callaghan, T. A.; Paul, D. R. Macromolecules 1993, 26, 2439-2450.

(28) Roe, R.-J. Methods of X-ray and Neutron Scattering in Polymer Science; Oxford University Press: New York, 1999.

(29) Winey, K. I.; Thomas, E. L.; Fetters, L. J. Macromolecules 1992, 25 , $422-428$.

(30) Kinning, D. J.; Winey, K. I.; Thomas, E. L. Macromolecules 1988, $21,3502-3506$

(31) Listak, J.; Bockstaller, M. R. Macromolecules 2006, 39, 5820-5825.

(32) Russell, T. P.; Lin, J. S.; Spooner, S.; Wignall, G. D. J. Appl. Crystallogr. 1988, 21, 628-538.

MA070447T 